\title{
Application of Run Length Encoding In Track Vision Inspection System
}

\author{
KANG FEI \\ Guidao jiaotong Polytechnic Institute, China \\ kangfei56@foxmail.com
}

\begin{abstract}
Keywords: run length encoding, morphological dilation, image processing, vision inspection Abstract. Optimized the binary image morphological dilation algorithm by run length encoding with the optimization features in background redundant and intersection and union simple operation, the Run Length Encoding-Morphological Dilation optimized algorithm had been proposed. The track contour binary image was processed by morphological dilation with the optimized algorithm. Compared analysis based on the image processing result and the speed before and after optimization. The edge burr and disconnection phenomenon can be removed by the optimized algorithm. The processing result of the track contour single frame image increased 5 times. The detection accuracy of the track visual inspection system can be improved to 20 points per meter.
\end{abstract}

\section{Introduction}

With the rapid development of Chinese railway construction, high-speed heavy has been the tendency. Vision solutions often used to check the track diseases in the conservation. The edge burr and disconnection phenomenon often caused in the image pre-processing and threshold segmentation by the Track visual inspection system. The mainly method to eliminate was the dilation process of morphological operation, but it took a large number of convolution operation. In this paper, morphological dilation process was optimized by run length encoding which not considered the background area and border region only described the core area and the internal characteristics. In addition, the simple operation of the intersection and union was another important advantage to the optimized morphological dilation effectively ${ }^{[1]}$. At present, the optimized algorithm of run-length encoding in morphological dilation has been proved efficiently in running memory and computing time $^{[2]}$. Trein $\mathrm{J}$ et al proposed a FPGA-based run-length encoding arithmetic logic circuit which realized the high-speed hardware execution and parallel input of image data. The optimized algorithm of run-length encoding could effectively improve the speed of image preprocessing, and easy to implement on the hardware for high speed processing.

In this paper, run-length encoding was used to optimize the morphological dilation algorithm, and applied in the image preprocessing of the Track Vision Inspection System. The basic principle and operation characteristics were analyzed and discussed the computing process of the optimized algorithm. Finally the improvement process of the image preprocessing would be verified by theoretical analysis and experimental verification.

\section{Principle of the Track Vision Inspection System}

Traditional contact track inspection equipment was replaced by non-contact vision solution as high failure, poor rate of the detection accuracy and bad interference. Machine vision theory was used to extract the track cross-sectional geometry by Track Vision Inspection System with multi-sensor data fusion to correct the data. Fig. 1 is a schematic diagram of Track Vision Inspection System, which mainly composed with high-speed CCD camera and line laser. The original image data was identified by high-speed CCD camera from reflection imaging generated by laser, then computed the pixel outline of the track cross section through a series of algorithms. 


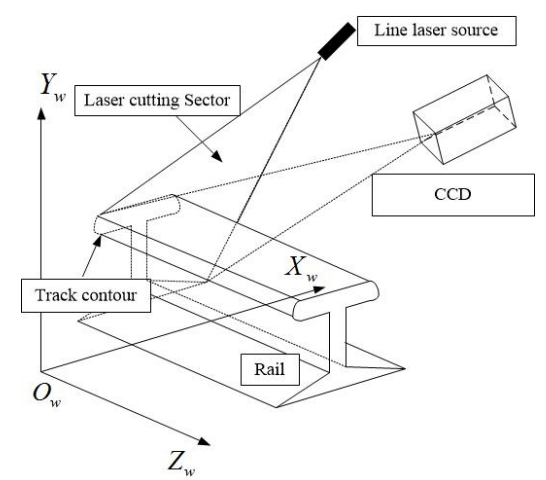

Fig. 1 Schematic diagram of Track Vision Inspection System

As the image data is a huge amount of information, speeding of the image processing algorithm was the key of the detection performance of the whole system. Image preprocessing was used for inhibiting invalidity information, enhancing effective features, which occupied most of the frame processing time and operational memory ${ }^{[3]}$. So the key to improve the image processing speed was the optimization of image preprocessing.

\section{Run Length Encoding-Morphological Dilation optimized algorithm}

Run-length encoding is used to describe the core area of the image, pixels with the same gray scale value sequence made up a run-length in a progressive scan image matrix. The run-length only stores the gray constant value $\mathrm{k}$ and the length $\mathrm{L}$, which avoiding repeated storage of the same gray value. If a gray scale of $\alpha$ row in a $n \times n$ area image data matrix were $\left(k_{0}, k_{1} \ldots \ldots k_{n}\right), k_{i} \sim k_{i+5}$ among them were a pixel aggregate, the gray constant value was $k$, then run-length encode was $(k, 6)$.

By definition the ROI (Region of Interest) could be seen as a collection of run-length encode. Therefore, it could avoid a lot of duplication of redundant computing in logic operation for intersection and union.

Due to the interference of light, it often appeared edge burr and disconnection phenomenon after threshold segmentation processing during the process of track vision inspection, which had seriously affected the accuracy of the system detected. So the morphological dilation was used to regrow the burr and disconnection edge.

Definition of the morphological dilation as Eq.1 shows:

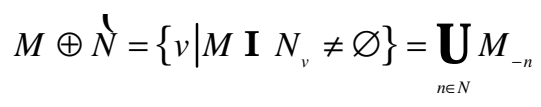

Among them, $\mathrm{M}$ represents ROI, $\mathrm{N}$ represents the structural elements.

The morphological dilation can be considered using a transpose structural element in Minkowski Addition. The Minkowski addition can be defined as Eq.2 shows ${ }^{[4]}$ :

$$
\begin{aligned}
M \oplus N & =\{m+n \mid m \in M, n \in N\} \\
& =\left\{v|M|(N)_{v} \neq \varnothing\right\}
\end{aligned}
$$

By definition it meet the commutative law as $M \oplus N=N \oplus M$ and the distributive law as $(M \cup N) \oplus T=M \oplus T \cup N \oplus T$, which is an important foundation for the establishment of the Run Length Encoding-Morphological Dilation optimized algorithm.

If $m_{i}, n_{j}$ are respectively a single run-length encoding of ROI and structural elements, $a$ and $b$ represented the number of run length for each of the two regions. The specific process of the Run Length Encoding-Morphological Dilation optimized algorithm as follows: 
First of all, represented ROI and structural elements respectively by run-length encoding with $\bigcup_{i=1}^{a} m_{i}$ and $\bigcup_{j=1}^{b} n_{j}$.

Point by point sought a non-empty intersection with ROI and structural element, which was converted into using run-length encoding to solve as Eq.3 shows:

$$
M \oplus N=\left(\bigcup_{i=1}^{a} m_{i}\right) \oplus\left(\bigcup_{j=1}^{b} n_{j}\right)
$$

Calculated the result of morphological dilation for single run length $\mathrm{m}_{\mathrm{i}}, \mathrm{n}_{\mathrm{j}}$ as $m_{i} \oplus n_{j}$, then seeking union set of $\mathrm{a} \times \mathrm{b}$ times single run length results, The optimized Minkowski addition could be represented by run-length encoding as Eq. 4 shows:

$$
M \oplus N=\bigcup_{i=1}^{a} \bigcup_{j=1}^{b} m_{i} \oplus n_{j}
$$

Time complexity $T(\mathrm{n})=\mathrm{O}(f(\mathrm{n}))$ was used to operational efficiency comparison before and after optimization algorithm. Growth rate of the algorithm execution time was proportional to the complexity of the function $f(\mathrm{n})$.If the size of the rectangular image and ROI were $\mathbf{U} \times \mathbf{U}$ and $\mathbf{u} \times \mathbf{u}$ pixels, then the run length were $U$ and $u$. The structural element was a circular area with the diameter of $v$. The time complexity before optimization was $\mathrm{O}\left(\mathrm{u}^{2} \mathrm{v}^{2}\right)$, which was $\mathrm{O}(\mathrm{uv})$ optimized. The efficiency of the algorithm increased by secondary power. The amount of calculation significantly decreased.

In summary, the Run Length Encoding-Morphological Dilation optimized algorithm could be used in the Track Vision Inspection System which was highly demanding in real-time image processing. In addition, the interference could not be completely eliminated by morphological dilation in practical application process. Filtering and gradation transformation were necessary to reduce the light spot noise $^{[5]}$.

\section{Experimental Verification and data analysis}

Fig.2 was an $800 \times 640$ 8-bit track contour image extracted by the Track Vision Inspection System. Experimental conditions as follows: fluorescent lamp diffuse reflectance lighting, the CCD pixel size was $5.6 \mu \mathrm{m} \times 5.6 \mu \mathrm{m}$, the frame rate was $60 \mathrm{fps}, \mathrm{SNR}>52 \mathrm{~dB}$. The white bright band generated by linear laser light sources which vertical irradiated track. The laser light source was red which had a $10^{\circ}$ fan angle and a wavelength of $650 \mathrm{~nm}$.

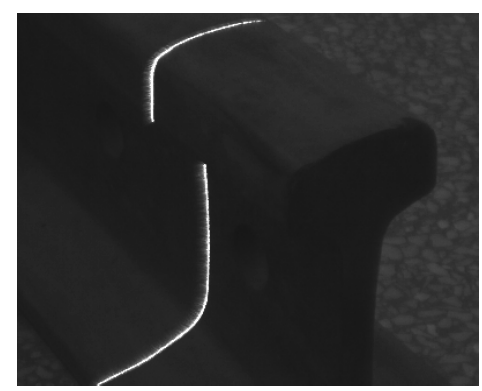

Fig. 2 Track Image

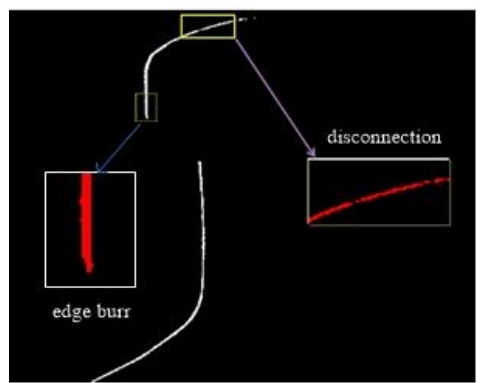

Fig.3 Disconnection and edge burr

The experiment result of image threshold segmentation were shown in Fig.3, there were disconnection and edge burr when the binary image was enlarged. These phenomenon would lead to inaccurate pixel coordinates in further refine process. In order to address this problem, morphological dilation was used for binary image after threshold segmentation. Run Length Encoding-Morphological Dilation optimized algorithm was used and the results as Fig. 4. 


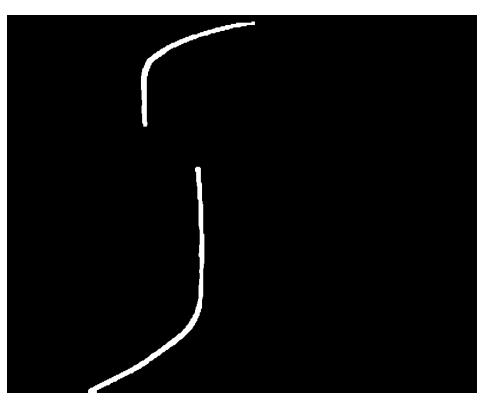

Fig. 4 Optimization algorithm results

Disconnection and edge burr were eliminated by optimization, then obtained a smooth contour of the track, formed a complete communication area. From analysis of algorithm, it did not change the results of the morphological dilation, which only optimized the calculation process.

Compared before and after of image processing time of 10 images for further verification. The statistics results were shown in Table 1.

Table 1 The image processing time statistic of 10 images before and after optimization [ms]

\begin{tabular}{|c|c|c|c|c|c|}
\hline NO. & 1 & 2 & 3 & 4 & 5 \\
\hline $\begin{array}{l}\text { before the } \\
\text { optimization }\end{array}$ & 124 & 119 & 123 & 124 & 122 \\
\hline $\begin{array}{l}\text { after the } \\
\text { optimization }\end{array}$ & 26 & 24 & 22 & 25 & 25 \\
\hline NO. & 6 & 7 & 8 & 9 & 10 \\
\hline $\begin{array}{l}\text { before the } \\
\text { optimization }\end{array}$ & 119 & 122 & 121 & 118 & 125 \\
\hline $\begin{array}{l}\text { after the } \\
\text { optimization }\end{array}$ & 23 & 27 & 21 & 23 & 27 \\
\hline
\end{tabular}

Table 1 shows that the Run Length Encoding-Morphological Dilation optimized algorithm has a greater advantage in single frame image processing speed. For the further exploration of the optimization result in different pixel size of the image, 100 frames of image were selected which were the same scene as Fig. 2 but with different pixel and processed, The relation of the average frame processing time before and after optimization and image pixel size were shown in Fig.5.

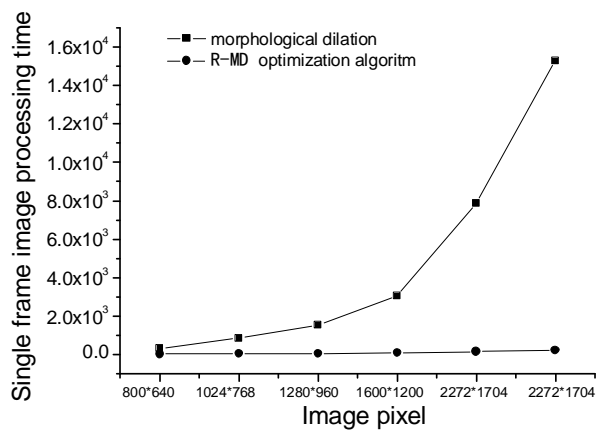

Fig.5 The average frame processing time before and after optimization with different pixel image

The Fig.5 shows that the average frame processing time of binary image dilation processing is increased with image pixels, but the optimization algorithm can solve this problem well. Therefore it can completely meet the real time inspection requirements of the Track Vision Inspection System. 


\section{Summary}

In this paper, Run Length Encoding-Morphological Dilation optimized algorithm was studied to solve the edge burrs and disconnection phenomenon in process of track contour image threshold segmentation. The preprocessing speed difference had been analyzed before and after the optimized image preprocessing. The experimental result showed that the processing speed of the optimization increased by 5 times than the direct dilation process. The detection accuracy of the track visual inspection system can be improved to 20 points per meter.

\section{References}

[1] Sonka M, Hlavac V, Boyle R. Image processing, analysis and machine vision[M]. Thomson-Engineering, 2007.

[2] Wook-Joong Kim, Seong-Dae Kim, Kyuheon Kim. Fast algorithms for binary dilation and erosion using run-length encoding[J]. ETRI journal. 2005, 27(6): 814-817.

[3] Min Y Z, Dang J W, Zhang Z H. Online Surface Settlement Monitoring System of Ballastless Track with Difference Image Style[C]//Applied Mechanics and Materials. 2013, 367: 259-263.

[4] Carsten Steger, Markus Ulrich, Christian Wiedemann. Machine vision algorithms and applications[M]. wiley-VCH, 2008.

[5] Resendiz E.,Hart J. M.,Ahuja N..Automated visual inspection of railroad tracks[J].Intelligent Transportation Systems,2013,14(2):751-760. 